\title{
Split fermionic WIMPs evade direct detection
}

\author{
Karim Ghorbani \\ Physics Department, Faculty of Sciences, Arak University, \\ Arak 38156-8-8349, Iran \\ E-mail: karim1.ghorbani@gmail.com
}

ABSTRACT: We consider a model with two gauge singlet fermionic WIMPs communicating with the SM particles by a singlet scalar mediator via a Higgs portal. While the light WIMP is stable and plays the role of the dark matter (DM) candidate, the heavy partner is a short-lived WIMP without contribution to the current DM relic density. Along with the coannihilation effects the heavy WIMP, acting as a mediator in $t$ - and $u$-channel DM annihilation cross sections, has a significant effect in finding the viable parameter space against the direct detection constraints provided by XENON1t and LUX experiments. This is an extension to the minimal singlet fermionic DM model whose entire parameter space (except a resonance region) excluded by the latest direct detection experiments. It is found out that there are viable regions in the parameter space which evade direct detection upper bounds and respect the observed DM relic density by WMAP/Planck. We also found that the Fermi-LAT upper limits on the DM annihilation cross section into $b \bar{b}$ can exclude small regions of the viable parameter space which elude direct detection experiments. This model exemplifies a case within the WIMP paradigm whose DM candidate can escape direct detection experiments nontrivially. Such models are interesting to be studied in collider experiments like the LHC.

KEywords: Cosmology of Theories beyond the SM, Beyond Standard Model

ARXIV EPRINT: 1805.02098 


\section{Contents}

1 Introduction 1

2 The model 2

3 Lifetime of the heavy WIMP 3

4 Thermal relic density and coannihilation effects 4

5 Direct detection $\quad 6$

6 Indirect detection $\quad 11$

7 Conclusions $\quad 12$

A Annihilation cross sections $\quad 12$

\section{Introduction}

One of the underlying questions in astro-particle physics is about the nature of dark matter (DM). The current evidence for the existence of DM is ample and all of them are inferred from DM gravitational effects $[1,2]$. The standard model (SM) of particle physics has been remarkably successful in many aspects but concerning the nature of DM, it is doomed to failure. Although the present value of the DM relic density is measured with good precision by WMAP [3] and Planck [4], we do not know yet the type and strength of the fundamental interactions of DM with the baryonic matter.

So far, the current dedicated direct detection experiments have not manifested any sign of DM interaction with ordinary matter. However, the latest results by XENON1t [5] and LUX $[6,7]$ for the DM-nucleon spin-independent/dependent cross section provide us with the stringent upper limits on the DM-nucleon cross sections for DM mass in the range of $\sim 10 \mathrm{GeV}$ up to $\sim$ a few TeV. The experimental upper bounds on the cross section can exclude or at least constrain various DM models which are attempting to explain the observed DM relic density.

One popular and noted paradigm for the DM is the weakly interacting massive particles (WIMP) produced via freeze-out mechanism [8] where DM candidate has weak-scale interaction with the SM particles. In typical WIMP models, new elementary or composite particles play the role of DM candidate. Since the beginning of the construction of the WIMP models, the interaction of DM with normal matter through the SM Higgs portal has been an appealing possibility. The discovery of the Higgs boson [9, 10] reinforced the idea of the Higgs portal DM models. The Higgs discovery has been so welcome but its 
unnatural small mass uncovers the Higgs naturalness problem, for an interesting read in this regards one can consult [11]. One might then entertain the WIMP paradigm as a weak-scale solution to the Higgs naturalness problem as well, see for instance [12-14].

In building the WIMP models, the most minimal extensions to the SM look rationale to begin with. For a recent review on the various DM simplified models see [15] and references therein. The singlet scalar DM model [16-18] and the singlet fermionic DM model [19-21] exemplify the most minimal renormalizable WIMP models. The direct detection experiments have reached an unprecedented upper limits on the cross section, such that we witness today that the most minimal DM models $[18,19]$ are getting excluded partially in the case of scalar DM [22] or entirely in the case of fermionic DM [23]. In [24] it is demonstrated that with two real scalar WIMPs coupled to the Higgs doublet, a viable DM mass above the Higgs resonance up to $\sim 200 \mathrm{GeV}$ is accessible. Some nonminimal extensions to the SM in order to save the singlet scalar DM are studied in $[25,26]$.

In the present study, we extend the earlier work with a singlet fermionic DM [19] to a model with two singlet fermion WIMPs $\left(\psi_{1}\right.$ and $\left.\psi_{2}\right)$, where the light one is stable and thus plays the role of the DM candidate. The heavy WIMP is sufficiently short-lived with no contribution to the DM relic density. A singlet scalar, $\phi$, acting as a mediator makes the DM-Higgs interaction possible. Due to a new interaction term $\sim g_{12} \bar{\psi}_{1} \psi_{2}+$ (h.c) in our model, the model possesses an interesting characteristic even in the limit of large WIMP mass splitting. It is found that adding one more Dirac fermion field can change the viable parameter space nontrivially. It is changed in such a way that the DM candidate can leave the detector without leaving any traces in the detector.

The outline of the paper is as follows. In the next section, our DM model described. Lifetime of the heavy WIMP is evaluated numerically in section 3. Numerical analysis on the DM relic density and the coannihilation effects are given in section 4 . The main results of this study obtained in section 5 by imposing direct detection bounds. We finish with a conclusion. In the appendix, DM annihilation cross section formulas with two particles in the final state are provided.

\section{The model}

In this section we introduce a model as a minimal extension to the SM which contains two gauge singlet Dirac fermions $\left(\psi_{1}, \psi_{2}\right)$ one of which plays the role of the DM candidate, and a real singlet scalar $(\phi)$ as a mediator with the Lagrangian,

$$
\mathcal{L}_{\mathrm{DM}}=\bar{\psi}_{1}\left(i \not \partial-m_{1}\right) \psi_{1}+\bar{\psi}_{2}\left(i \not \partial-m_{2}\right) \psi_{2}+g_{1} \phi \bar{\psi}_{1} \psi_{1}+g_{2} \phi \bar{\psi}_{2} \psi_{2}+\left(g_{12} \phi \bar{\psi}_{1} \psi_{2}+\text { h.c. }\right) \text {. }
$$

The part of the Lagrangian which incorporates the new singlet scalar and the SM Higgs doublet is,

$$
\mathcal{L}(H, \phi)=\frac{1}{2}\left(\partial_{\mu} \phi\right)^{2}-\frac{m^{2}}{2} \phi^{2}-\frac{\lambda_{4}}{24} \phi^{4}-\frac{\lambda_{1}}{2} \phi H^{\dagger} H-\frac{\lambda_{2}}{2} \phi^{2} H^{\dagger} H+\mu_{H}^{2} H^{\dagger} H-\frac{\lambda_{H}}{4}\left(H^{\dagger} H\right)^{2} .
$$

As it is evident from the Lagrangians above, the only way for DM particle to interact with the SM particles is via a Higgs portal. Any other type of DM couplings to the SM particles 
are assumed to be negligible. It is well known that the Higgs doublet develops a non-zero vacuum expectation value (vev) as $v_{0}=246 \mathrm{GeV}$, and we make an innocuous assumption where the singlet scalar acquires a zero vev. In the unitary gauge we write down the Higgs doublet as $H=\left(0\left(v_{0}+h^{\prime}\right) / \sqrt{2}\right)^{T}$. The mass matrix for the scalars is not diagonal due to the term linear in the $\phi$ field. The mass matrix at tree level reads

$$
\mathbf{M}^{2}=\left(\begin{array}{cc}
M_{h^{\prime}}^{2} & \frac{1}{2} M_{\phi h^{\prime}}^{2} \\
\frac{1}{2} M_{\phi h^{\prime}}^{2} & M_{\phi}^{2}
\end{array}\right),
$$

where,

$$
M_{h^{\prime}}^{2}=\frac{1}{2} \lambda_{H} v_{0}^{2}, \quad M_{\phi h^{\prime}}^{2}=\lambda_{1} v_{0}, \quad M_{\phi}^{2}=m^{2}+\frac{1}{2} \lambda_{2} v_{0} .
$$

To find the mass eigenstates we need to diagonalize the mass matrix and this can be achieved by rotating the relevant fields appropriately,

$$
h=\sin (\theta) \phi+\cos (\theta) h^{\prime}, \quad s=\cos (\theta) \phi-\sin (\theta) h^{\prime},
$$

such that the mixing angle $\theta$ satisfies the relation,

$$
\tan 2 \theta=y, \quad y=\frac{2 M_{\phi h}^{2}}{M_{h}^{2}-M_{\phi}^{2}} .
$$

The mass eigenvalues are obtained as the following,

$$
m_{h, s}^{2}=\frac{M_{h^{\prime}}^{2}+M_{\phi}^{2}}{2} \pm \frac{M_{h^{\prime}}^{2}-M_{\phi}^{2}}{2} \sqrt{1+y^{2}}
$$

in which we choose $m_{h}=m_{H}$ for the SM Higgs mass with its measured value $125 \mathrm{GeV}$, and $m_{s}$ for the physical mass of the singlet scalar as a free parameter. It is possible to obtain two couplings in terms of the mixing angle and the physical masses of the scalars,

$$
\lambda_{1}=\frac{\sin (2 \theta)}{2 v_{0}}\left(m_{s}^{2}-m_{h}^{2}\right), \quad \lambda_{H}=\frac{m_{s}^{2} \sin ^{2}(\theta)+m_{h}^{2} \cos ^{2}(\theta)}{v_{0}^{2} / 2} .
$$

We assume that $m_{1}<m_{2}$, therefore $\psi_{1}$ is the stable fermion and the DM candidate, and $\psi_{2}$ is its heavier partner, hence, we set $m_{\mathrm{DM}}=m_{1}$. The mass splitting of the fermions is defined as $\Delta=m_{2}-m_{1}$. As a set of independent free parameters in the model we take $m_{s}, m_{1}, \Delta, \theta, g_{1}, g_{2}, g_{12}, \lambda_{2}$, and $\lambda_{4}$. Since our computations in this work are performed at tree level in perturbation theory, the coupling $\lambda_{4}$ does not come into play. We are then left with eight free parameters. The stability of the potential demands $\lambda_{4}>0, \lambda_{H}>0$ and in case $\lambda_{2}<0$ then we should have $\lambda_{4} \lambda_{H}>6 \lambda_{2}^{2}$.

\section{Lifetime of the heavy WIMP}

The minimal model presents two fermionic WIMPs, where its heavy component can decay into the light partner (DM) and the SM Higgs as $\psi_{2} \rightarrow \psi_{1} h$. Depending on the size of the mass splitting the SM Higgs may decay successively as $h \rightarrow \bar{f} f, W^{+} W^{-}, Z Z$, ss, where 


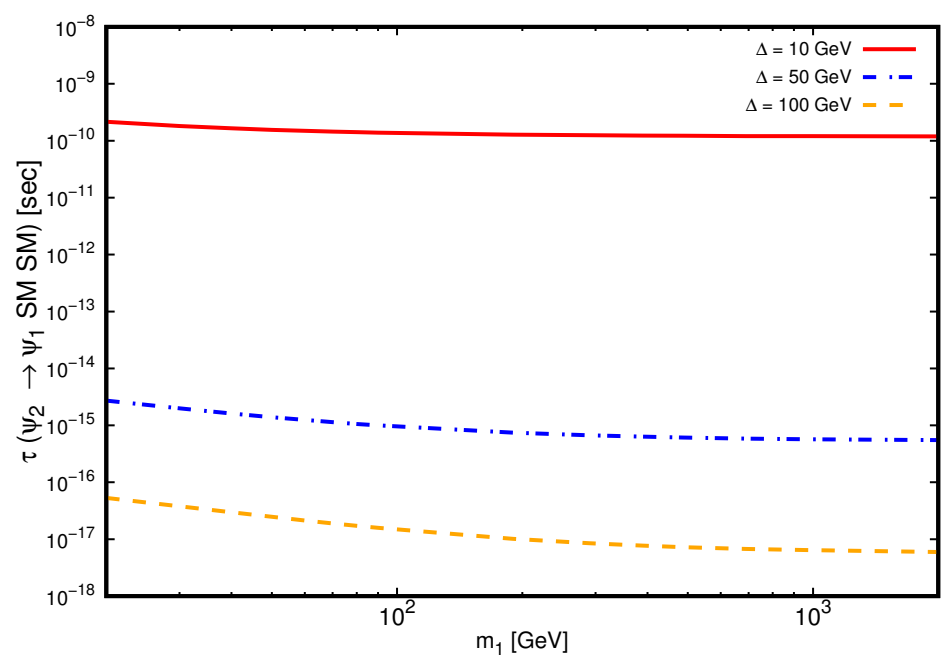

Figure 1. The lifetime of the heavy fermion is provided for three values of the mass splitting, $\Delta=10,50,100 \mathrm{GeV}$. Chosen input parameters are: $g_{12}=1, \sin \theta=0.05$ and $m_{s}=300 \mathrm{GeV}$.

$f$ stands for the SM fermions. If the mass splitting is assumed to be $\Delta<m_{H}$, then the intermediate Higgs is off-shell and its kinematically allowed modes are decays into a pair of leptons or quarks (excluding the top quark).

In case the lifetime of the heavy fermion is much less than the age of the Universe, then it has no contribution to the present DM relic density. It is therefore necessary to see this condition is fulfilled over the viable parameter space. To this end, we give an expression for the decay width of the heavy fermion. Given the assigned momenta to the particles in the decay as $\psi_{2}\left(p_{2}\right) \rightarrow \psi_{1}\left(p_{1}\right) \bar{f}\left(p_{3}\right) f\left(p_{4}\right)$, it reads

$$
\begin{gathered}
\Gamma=\iint d t d u \frac{N_{c} \sin ^{2} 2 \theta g_{12}^{2} m_{f}^{2}}{256 \pi^{3} m_{2}^{3} v_{h}^{2}}\left[2 m_{1} m_{2}\left(m_{1}^{2}+m_{2}^{2}\right)-\left(m_{1}^{2}+m_{2}^{2}\right)^{2}-t m_{1} m_{2}+2 m_{f}^{2}\left(\Delta^{2}-t\right)\right. \\
\left.+\frac{3}{2} t\left(m_{1}^{2}+m_{2}^{2}\right)-\frac{1}{2} t^{2}\right] \times\left|\frac{1}{\left(t-m_{s}^{2}\right)+i \Gamma_{s} m_{s}}-\frac{1}{\left(t-m_{h}^{2}\right)+i \Gamma_{h} m_{h}}\right|^{2}
\end{gathered}
$$

where $N_{c}$ is the number of color for the SM fermion, and the Mandelstam variables are defined as $t=\left(p_{3}+p_{4}\right)^{2}$ and $u=\left(p_{2}+p_{3}\right)^{2}$. The decay rate of the heavy fermion is then $\tau=1 / \Gamma$. We compute the decay rate numerically utilizing the program CalcHEP [27] for three distinct values of the mass splitting, $\Delta=10,50$ and $100 \mathrm{GeV}$. The relevant input parameters are chosen as $g_{12}=1$., $\sin \theta=0.05$ and $m_{s}=300 \mathrm{GeV}$. For the DM mass in the range $30 \mathrm{GeV}<m_{1}<2 \mathrm{TeV}$, it is apparent in figure 1 that the decay rate is much smaller than the age of the Universe and therefore has no contribution to the DM relic density.

\section{Thermal relic density and coannihilation effects}

One natural way to explain the present DM relic density within the WIMP paradigm, is the production of thermal relic. In this process, WIMP particle(s) is in thermal equilibrium in the early Universe but, at a specific temperature named freeze-out temperature, it leaves 

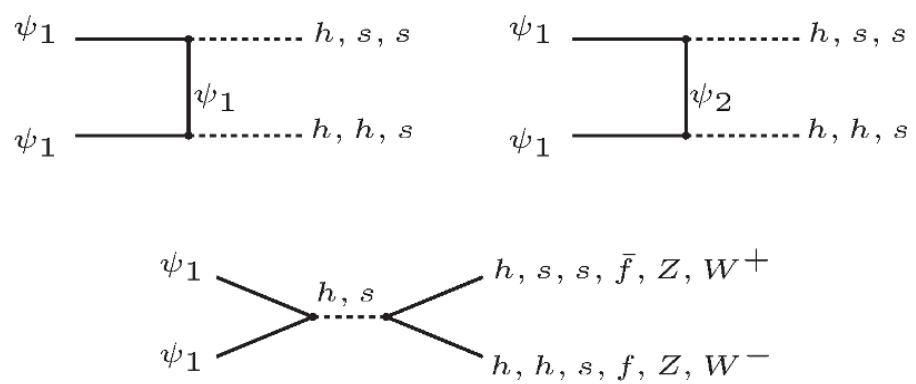

Figure 2. Feynman diagrams for the DM annihilation processes are shown. Only diagrams with two particles in the final state are given here.

the equilibrium and its density remains constant afterwards. The size of the relic density depends strongly on the freeze-out temperature, and the later depends in turn on the WIMPs annihilation cross sections in the early Universe.

In this work, we have a model with two participating WIMPs, in which the light one is our DM candidate. Besides the usual DM annihilation processes, there are events where DM candidate annihilates in tandem with another WIMP. This process is called coannihilation. In figure 2 all possible annihilation diagrams for the DM candidate with two particles in the final state are displayed. In the $s$-channel we have DM annihilation into $\bar{f} f, W^{+} W^{-}, Z Z, h h, h s, s s$ and in the $t$ - and $u$-channel we have annihilation into $h h, h s, s s$. If one replaces a field $\psi_{1}$ with a field $\psi_{2}$ in the first two diagrams in figure 2 then the coannihilation processes will be obtained.

In the case of two component WIMPs, to evaluate the DM relic abundance one needs to solve two coupled Boltzmann equations which give us the time evolution of the WIMPs number densities. In practice, it suffices to use a single Boltzmann equation with an effective (co)annihilation cross section instead of two Boltzmann equations [28, 29]. The resulting equation for the total number density, $n=n_{1}+n_{2}$, is

$$
\frac{d n}{d t}=-3 H n-\left\langle\sigma_{\mathrm{eff}} v\right\rangle\left(n^{2}-n_{e q}^{2}\right)
$$

in which $H$ is the Hubble constant and the effective cross section is given by

$$
\sigma_{\mathrm{eff}}=\frac{4}{g_{\mathrm{eff}}^{2}}\left(\sigma_{11}+\sigma_{22}\left(1+\frac{\Delta}{m_{1}}\right)^{3} e^{-2 \Delta / T}+2 \sigma_{12}\left(1+\frac{\Delta}{m_{1}}\right)^{3 / 2} e^{-\Delta / T}\right),
$$

where, $\sigma_{i j}$ denotes (co)annihilation cross section for the process, $\psi_{i} \psi_{j} \rightarrow \mathrm{SM}$. The effective number of internal degrees of freedom is $g_{\text {eff }}=2+2\left(1+\frac{\Delta}{m_{1}}\right)^{3 / 2} e^{-\Delta / T}$. In the Boltzmann equation, $\left\langle\sigma_{\text {eff }} v\right\rangle$ stands for the thermal averaged of the effective cross section times the relative DM velocity. In the appendix, formulas for $\sigma_{11}$ are given.

To obtain the DM relic density, one should solve the Boltzmann equation numerically. This is done in this work by exploiting the program MicrOMEGAs [30]. In this stage we would like to study the dependency of the DM relic density on the mass splitting $\Delta$ in terms of the DM mass. In figure 3 our results are shown for three values of the mass splitting, 


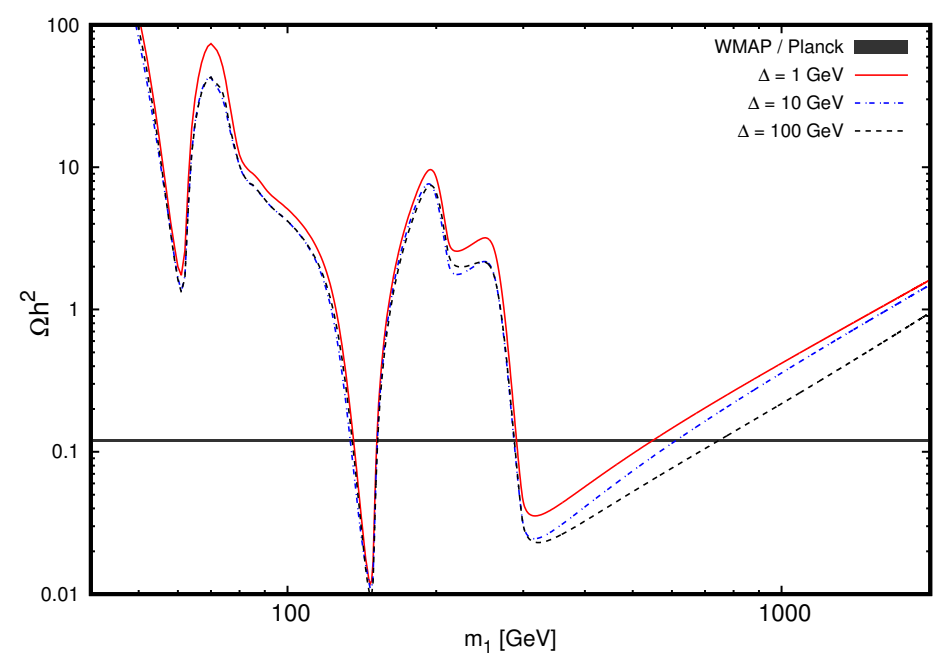

Figure 3. DM relic density is shown as a function of DM mass for three values of the mass splitting, $\Delta=1,10,100 \mathrm{GeV}$. Input parameters are: $g_{1}=0.9, g_{2}=0.1, g_{12}=0.9, \lambda_{2}=0.1, \sin \theta=0.05$ and $m_{s}=300 \mathrm{GeV}$.

$\Delta=1,10,100 \mathrm{GeV}$. The parameters used as input are, $g_{1}=0.9, g_{2}=0.1, g_{12}=0.9$, $\lambda_{2}=0.1, \sin \theta=0.05$ and $m_{s}=300 \mathrm{GeV}$.

It is evident from the plots in figure 3 that the coannihilation effects which are relevant for small $\Delta$, increase the DM relic density slightly. The same behavior for the coannihilation effects is found within the supersymmetric models in [31,32]. This can be compared with the case of scalar DM in the split-scalar model therein the relic density is decreased by the coannihilation effects [24]. It is also seen that in the resonance region the coannihilation effects are essentially so small since in this region the DM annihilation cross section, $\sigma_{11}$, dominates the effective cross section.

\section{Direct detection}

Our main results concerning the direct detection bounds on the model parameter space are discussed in this section. Direct detection experiments are set up in the hope that the strength of DM particle interaction with the nucleon is high enough such that finding DM footprints in the detector is feasible. Recent results from XENON1t and LUX experiments suggest that we are not able to find a DM signal in the detector yet. However, the most stringent exclusion limits are granted by these experiments.

Let us now find regions in our model parameter space where the DM candidate can evade the exclusion bounds. In order to find the DM-nucleon elastic scattering cross section, first one needs to have the DM interaction with ordinary matter in the quark level. The DM-quark interaction is possible in the present model by exchanging a SM Higgs or a singlet scalar via $t$-channel processes. In the limit of low momentum transfer, the relevant interaction can be described by an effective Lagrangian,

$$
\mathcal{L}_{\text {eff }}=c_{\mathrm{q}} \bar{\psi}_{1} \psi_{1} \bar{q} q
$$


with the effective coupling,

$$
c_{\mathrm{q}}=\left(g_{1} \sin 2 \theta\right) \frac{m_{\mathrm{q}}}{2 v_{0}}\left(\frac{1}{m_{h}^{2}}-\frac{1}{m_{s}^{2}}\right) .
$$

To promote the effective Lagrangian to the nucleon level, we appeal to the approximation at the very small momentum transfer, where a quark level matrix element can be replaced by a nucleonic level matrix element introducing a proportionality form factor [33, 34]. Therefore, we can ultimately obtain the spin-independent (SI) cross section for the DM-nucleon as

$$
\sigma_{\mathrm{SI}}^{\mathrm{N}}=\frac{4 \mu_{N}^{2} c_{N}^{2}}{\pi}
$$

where the reduced mass of the DM-nucleon is denoted by $\mu_{N}$ and, the coupling $c_{N}$ is given in terms of the quark effective coupling $c_{q}$ and scalar form factors as

$$
c_{N}=m_{N} \sum_{q=u, d, s} f_{T q}^{N} \frac{c_{q}}{m_{q}}+\frac{2}{27} f_{T g}^{N} \sum_{q=c, b, t} \frac{c_{q}}{m_{q}} .
$$

In the analysis to come, for the DM-proton elastic cross section we have used the scalar form factors below

$$
f_{u}^{p}=0.0153, \quad f_{d}^{p}=0.0191, \quad f_{s}^{p}=0.0447 .
$$

In the following numerical computations we always choose for the mixing angle $\sin \theta=0.05$, and for the singlet scalar we select two distinct masses, $m_{s}=80,300 \mathrm{GeV}$.

To begin, let us take a large value for the mass splitting, $\Delta=100 \mathrm{GeV}$, where according to the standard lore we expect the coannihilation effects to be relevant up to the DM masses such that $\frac{\Delta}{m_{\mathrm{DM}}} \lesssim 10 \%$. At first sight it seems that the present model should reduce to the singlet fermionic model at the limit of large mass splitting.

To check this out, we consider two cases (a) $g_{12}=0$ and (b) $g_{12} \neq 0$, and then move on to compute the DM-proton elastic scattering cross section as a function of the DM mass in the range $30 \mathrm{GeV}<m_{1}<2 \mathrm{TeV}$. We set, for the first case $0<g_{1}, g_{2}<2$, and for the second case $-1<g_{1}, g_{2}, g_{12}<1$.

In figure 4 direct detection cross sections are shown for the two cases where only points consistent with the observed relic density are picked. In the case with $g_{12}=0$ the entire parameter space is excluded by direct detection bounds except the resonance region around $m_{1} \sim m_{s} / 2$. This is in agreement with the results in [23]. However, in case $g_{12} \neq 0$, besides the resonance region there are points in the parameter space in the range $300 \mathrm{GeV}$ $\lesssim m_{1} \lesssim 600 \mathrm{GeV}$ residing well below the exclusion bounds. Since the coannihilation effects are believed to be tiny for $\Delta=100 \mathrm{GeV}$ in the above mentioned mass range, a pertinent question to pose is that why in case $g_{12} \neq 0$ there are points in the parameter space which can evade exclusion bounds. To address this question we should first note that at the limit of large mass splitting the effective (co)annihilation cross section becomes $\sigma_{\text {eff }} \sim \sigma_{11}$. Therefore, according to the annihilation cross section formulas in the appendix, in case $g_{12}=0$ then we have $\sigma_{\text {eff }} \propto g_{1}^{2}$, and when $g_{12} \neq 0$ then the effective cross section depends 

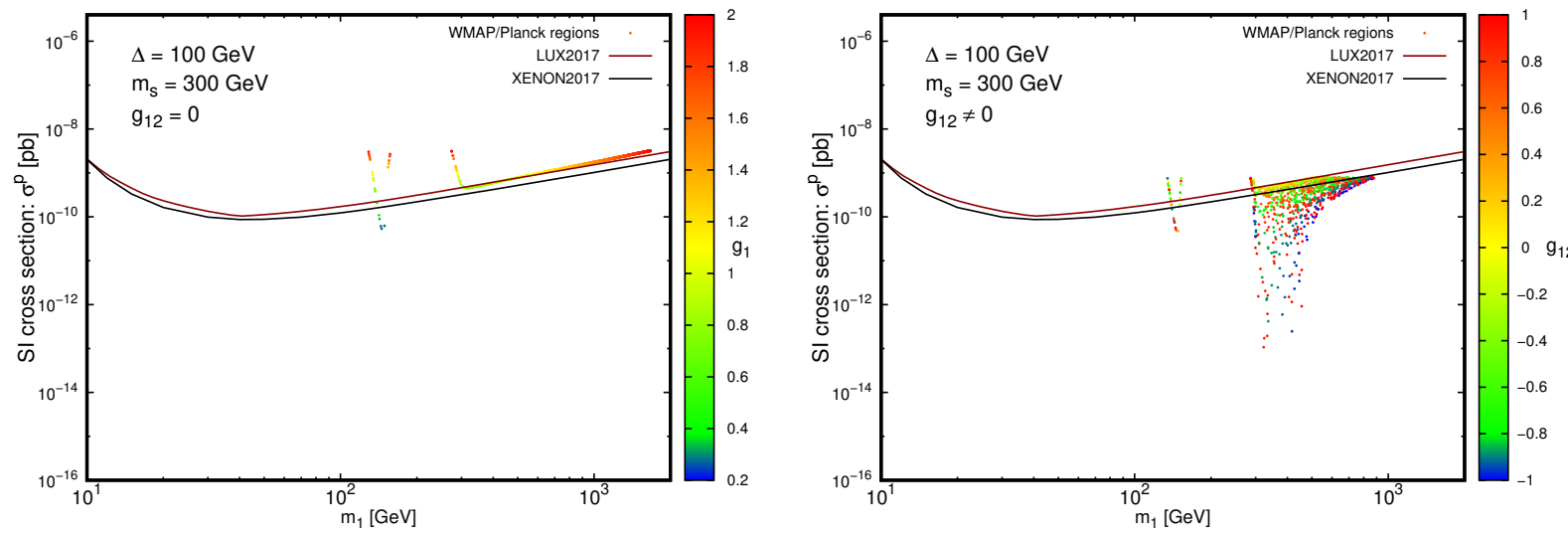

Figure 4. The DM-proton (SI) scattering cross section as a function of DM mass for the mass splitting $\Delta=100 \mathrm{GeV}$ and singlet scalar mass $m_{s}=300 \mathrm{GeV}$. In the left panel $g_{12}=0$ and in the right panel $g_{12} \neq 0$. The mixing angle is fixed at $\sin \theta=0.05$.

on the coupling $g_{1}, g_{12}$ in various ways, $\sigma_{\text {eff }} \propto g_{1}^{4}, \ldots, g_{12}^{4}$. On the other hand, the (SI) elastic scattering cross section, $\sigma_{\mathrm{SI}}^{\mathrm{N}}$, is proportional to $g_{1}^{2}$ as seen from the formulas in eq. (5.3) and eq. (5.4).

It is not difficult now to explain our results: when $g_{12}=0$, in order to get a large enough annihilation cross section (to induce the observed relic density) large value for the coupling $g_{1}$ is required. This in turn gives rise to a large (SI) elastic scattering cross section which is also proportional to $g_{1}^{2}$ and hence, the entire parameter space get excluded. When $g_{12} \neq 0$ it becomes possible to have a small (SI) elastic scattering cross section (small $g_{1}$ ) and at the same time large enough annihilation cross section, suitable to give the correct relic density. This is due to terms proportional to powers of the coupling $g_{12}$, necessitated to enhance the annihilation cross section.

Another important observation from figure 4 indicates that the viable DM mass when $m_{s}=300 \mathrm{GeV}$, starts from $m_{\mathrm{DM}} \sim 300$ up to higher masses for $g_{12} \neq 0$. The reason hinges on the fact that for the DM masses larger than $300 \mathrm{GeV}$, a new channel $\psi_{1} \bar{\psi}_{1} \rightarrow s s$ opens up for the DM annihilation which has dominant effects over the other already opened channels, namely, $\psi_{1} \bar{\psi}_{1} \rightarrow h h$ and $\psi_{1} \bar{\psi}_{1} \rightarrow s h$. In fact, when the process $\psi_{1} \bar{\psi}_{1} \rightarrow s s$ opens up, we get contributing terms proportional to $g_{1}^{2} g_{12}^{2} \cos ^{4} \theta$ or $g_{12}^{4} \cos ^{4} \theta$ while in the processes $\psi_{1} \bar{\psi}_{1} \rightarrow h h$ and $\psi_{1} \bar{\psi}_{1} \rightarrow h s$, the contributing terms to the new effect are proportional to $g_{1}^{2} g_{12}^{2} \sin ^{4} \theta$ or $g_{12}^{4} \sin ^{4} \theta$, and $g_{1}^{2} g_{12}^{2} \cos ^{2} \theta \sin ^{2} \theta$ or $g_{12}^{4} \cos ^{2} \theta \sin ^{2} \theta$, respectively. Since $\sin \theta=0.05$, it is easy to see that the process $\psi_{1} \bar{\psi}_{1} \rightarrow s s$ brings in the dominant effects and the DM mass threshold at about $300 \mathrm{GeV}$ becomes explicable.

For smaller mass splitting, $\Delta=1,10 \mathrm{GeV}$, the results are shown in figure 5 . The results indicate almost the same characteristic as the case with $\Delta=100 \mathrm{GeV}$.

In the next step, we redo our computations taking a smaller mass for the singlet scalar, i.e., $m_{s}=80 \mathrm{GeV}$ and the same values for the other input parameters. Our findings are given in figure 6 for $\Delta=1,10 \mathrm{GeV}$, and in figure 7 for $\Delta=100 \mathrm{GeV}$. The range of the viable DM mass is now broaden significantly, from $m_{1} \sim m_{s} / 2$ up to masses $\sim 600 \mathrm{GeV}$ in 

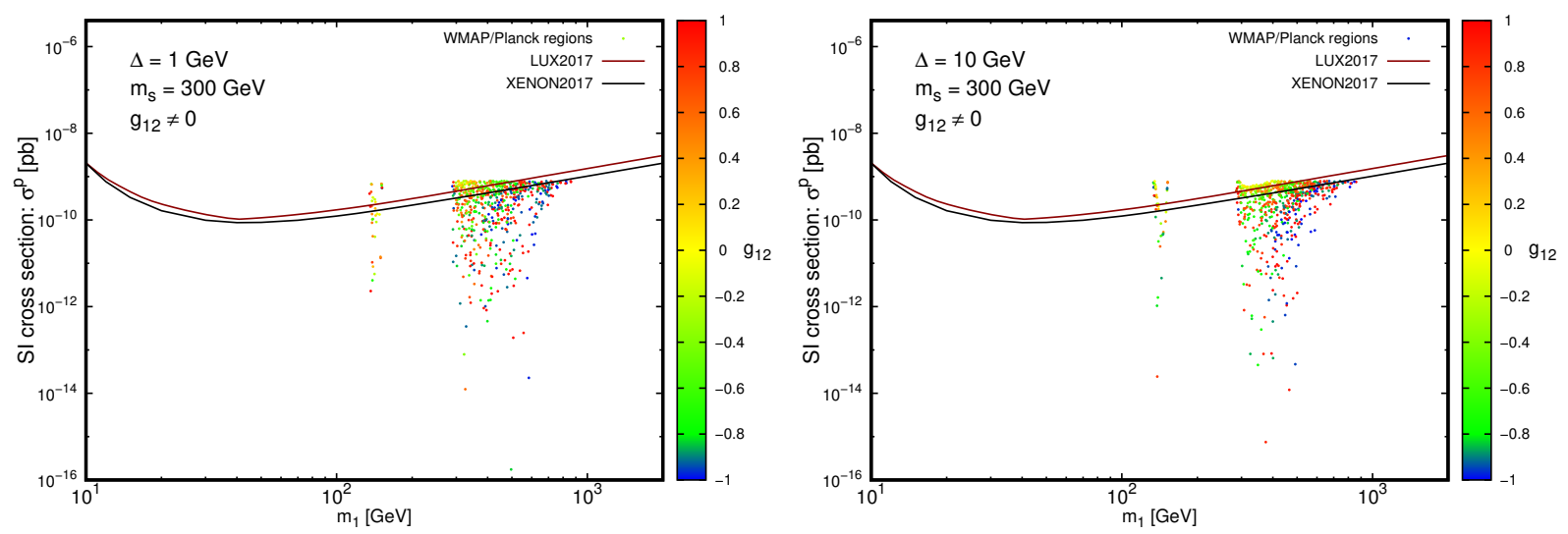

Figure 5. The DM-proton (SI) scattering cross section as a function of DM mass for the mass split$\operatorname{ting} \Delta=1,10 \mathrm{GeV}$. The singlet scalar mass is $m_{s}=300 \mathrm{GeV}$ and the mixing angle is $\sin \theta=0.05$.
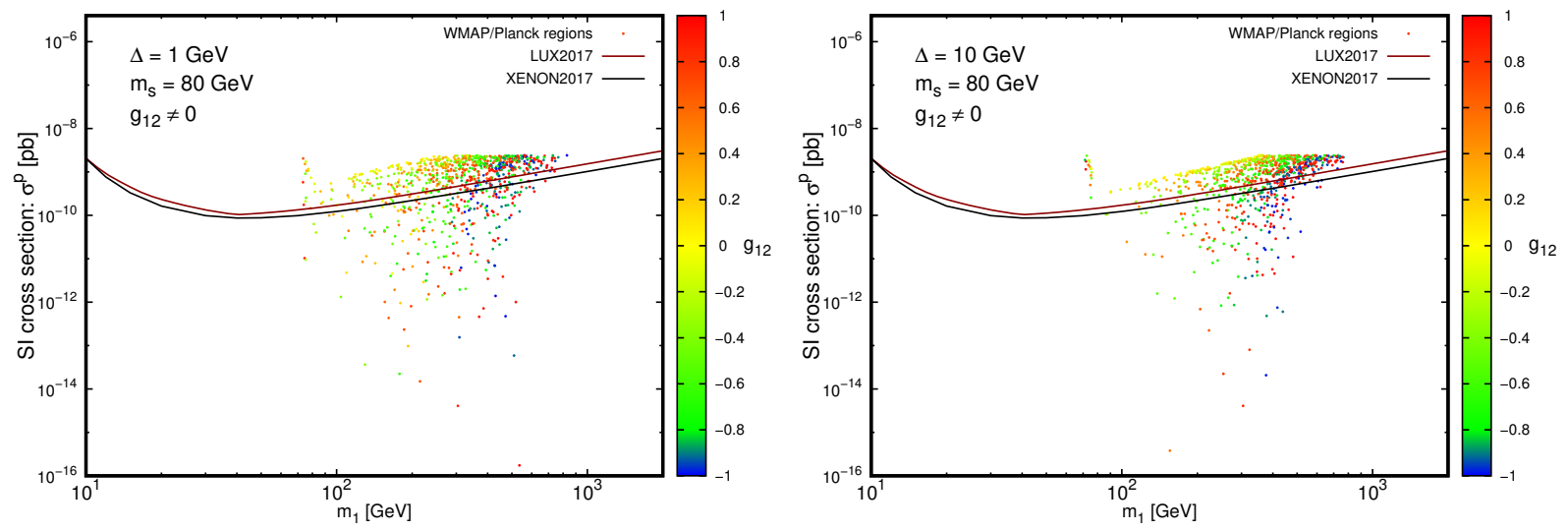

Figure 6. The DM-proton (SI) scattering cross section as a function of DM mass for the mass splitting $\Delta=1,10 \mathrm{GeV}$, and the singlet scalar mass $m_{s}=80 \mathrm{GeV}$.

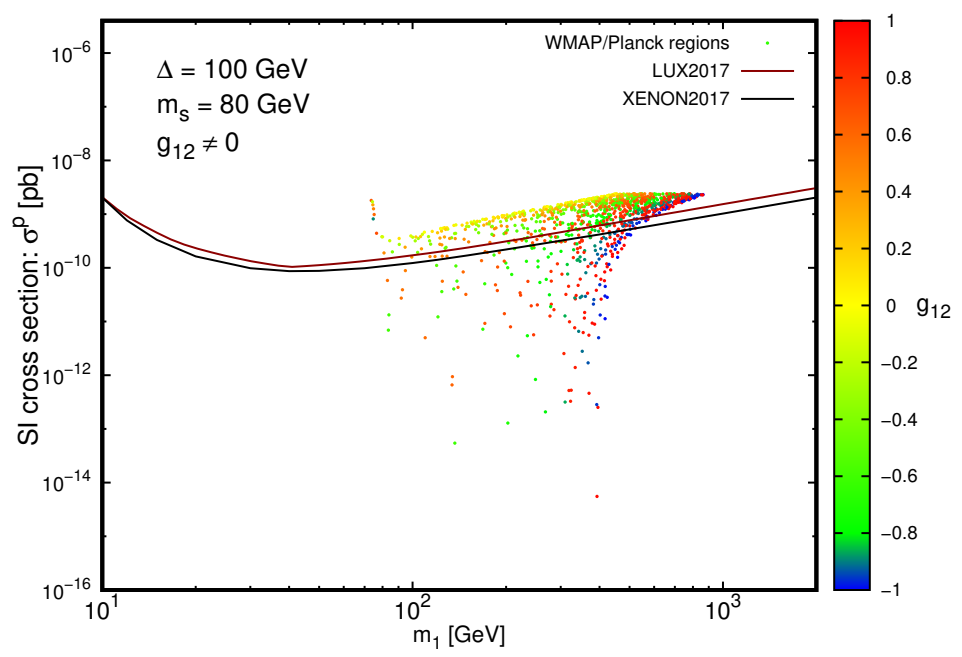

Figure 7. The DM-proton (SI) scattering cross section as a function of DM mass for the mass splitting $\Delta=100 \mathrm{GeV}$, and the singlet scalar mass $m_{s}=80 \mathrm{GeV}$. 

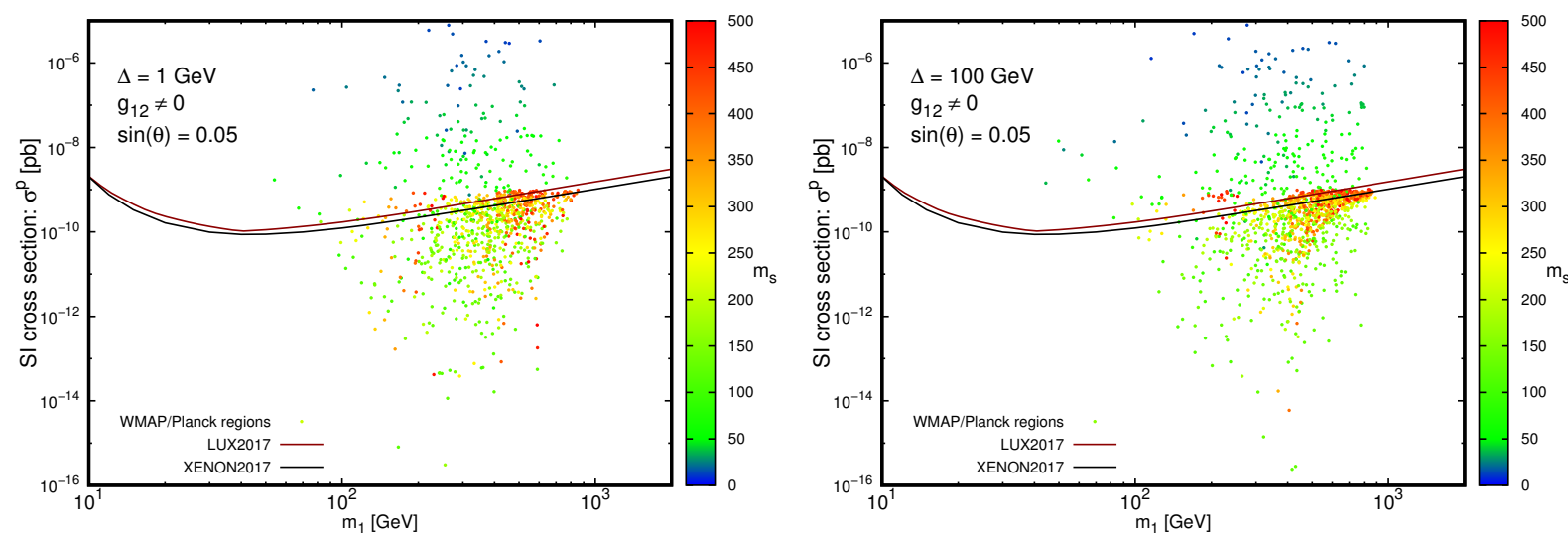

Figure 8. The DM-proton (SI) scattering cross section as a function of DM mass for the mass splitting $\Delta=1 \mathrm{GeV}$ (left) and $\Delta=100 \mathrm{GeV}$ (right). The singlet scalar mass is $10 \mathrm{GeV}<m_{s}<500 \mathrm{GeV}$ and the mixing angle is $\sin \theta=0.05$. The couplings are chosen as $-1<g_{1}, g_{2}, g_{12}<1$.
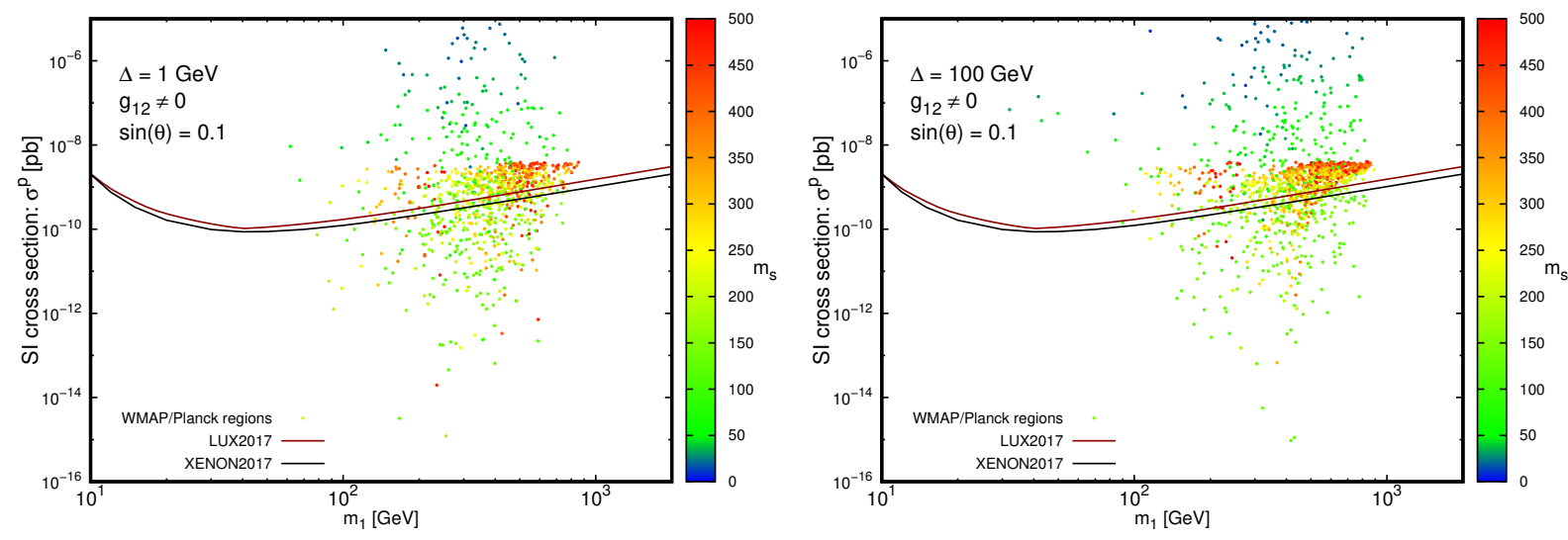

Figure 9. The DM-proton (SI) scattering cross section as a function of DM mass for the mass splitting $\Delta=1 \mathrm{GeV}$ (left) and $\Delta=100 \mathrm{GeV}$ (right). The singlet scalar mass is $10 \mathrm{GeV}<m_{s}<500 \mathrm{GeV}$ and the mixing angle is $\sin \theta=0.1$. The couplings are chosen as $-1<g_{1}, g_{2}, g_{12}<1$.

the case with $\Delta=1 \mathrm{GeV}$. At larger mass splitting that the coannihilation effects are less important the lower viable DM mass is pushed a little upward. Again, the same line of reasoning can be applied to explain the viable DM mass range.

Finally, we take the singlet scalar mass in the range $10 \mathrm{GeV}<m_{s}<500 \mathrm{GeV}$ and compute the DM-nucleon scattering cross section for two distinct values of the mass splitting $\Delta=1,100 \mathrm{GeV}$ and for two values of the mixing angle, $\sin \theta=0.05,0.1$. The regions of the parameter space respecting the observed relic density are shown in figure 8 with $\sin \theta=0.05$ and in figure 9 with $\sin \theta=0.1$. The results indicate that singlet scalar masses with $m_{s} \lesssim 30 \mathrm{GeV}$ are excluded by direct detection upper bounds irrespective of our choices for the mass splitting and the mixing angle. 

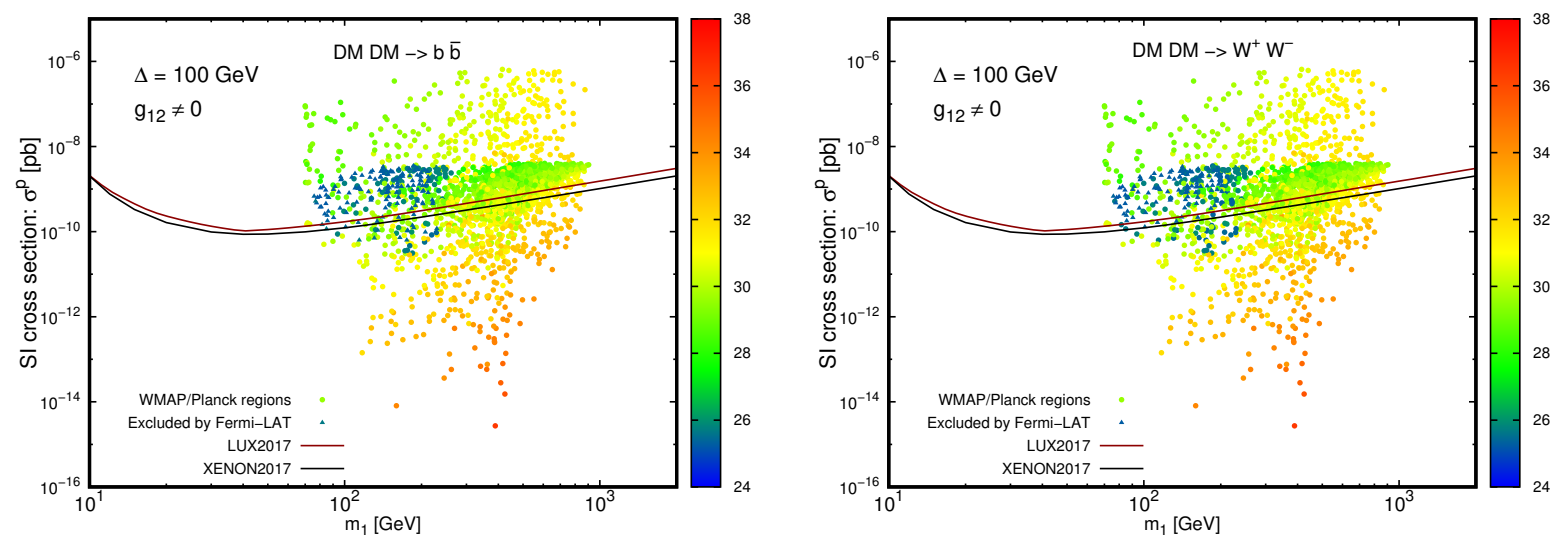

Figure 10. The DM-proton (SI) scattering cross section as a function of DM mass for the mass splitting $\Delta=100 \mathrm{GeV}$. The vertical color spectrum indicates the quantity $-\log _{10}\langle\sigma v\rangle$ for DM annihilation into $b \bar{b}$ (left) and DM annihilation into $W^{+} W^{-}$(right).

\section{Indirect detection}

The existence of DM in regions with high density like the Galactic Center and dwarf spheroidal satellite galaxies (dSphs) of the Milky Way might lead to self-annihilation of DM into energetic SM particles. This motivates indirect search for DM. Fermi Large Area Telescope (Fermi-LAT) collection of gamma-ray data for six years from Milky Way dSphs [35] and the H.E.S.S. (High Energy Stereoscopic System) ground-based cherenkov telescopes with ten years of Galactic Center gamma-ray data collection [36] do not indicate any significant gamma-ray excess.

However, H.E.S.S., assuming Einasto and NFW DM density profiles at the Galactic Center provides us with the upper bounds on the velocity-weighted annihilation cross section $\langle\sigma v\rangle$ for various channels, namely, annihilation into quark pair $(b \bar{b}, t \bar{t})$, gauge boson pair $\left(W^{+} W^{-}\right)$and lepton pair $\left(\mu^{+} \mu^{-}, \tau \bar{\tau}\right)$ channels. The strongest upper limit is obtained for a particle DM mass of about $1 \mathrm{TeV}$ in the $\tau^{+} \tau^{-}$channel at $\sim 2 \times 10^{-26} \mathrm{~cm}^{3} \mathrm{~s}^{-1}$ [36]. In addition, Fermi-LAT gives us the upper limits on the annihilation cross sections for the same channels including $e^{+} e^{-}$channel [35].

For the regions of the parameter space we are interested in the Fermi-LAT upper limits are slightly stronger, thus these limits are applied in our probe over the viable regions.

In our numerical computations we pick the free parameters as $\Delta=100 \mathrm{GeV}, \sin \theta=0.1$, $30 \mathrm{GeV}<m_{s}<500 \mathrm{GeV}, 10 \mathrm{GeV}<m_{1}<2000 \mathrm{GeV}$ and $-1<g_{i}<1$. In figure 10 our results are shown for the DM-proton cross section in terms of the DM mass, and also the quantity $-\log _{10}\langle\sigma v\rangle$ is given for the DM annihilation into $b \bar{b}$ and $W^{+} W^{-}$constrained by the Fermi-LAT upper limits. It is found that the Fermi-LAT constraints on $\langle\sigma v\rangle_{b \bar{b}}$ is slightly stronger than that on $\langle\sigma v\rangle_{W^{+} W^{-}}$, such that a small portion of the viable parameter space gets excluded by the Fermi-LAT upper limits on $\langle\sigma v\rangle_{b \bar{b}}$ but Fermi-LAT upper bounds on $\langle\sigma v\rangle_{W^{+} W^{-}}$can only exclude regions in the parameter space which is already excluded by direct detection experiments. We also checked and found that other channels probed by Fermi-LAT i.e., $e^{+} e^{-}, \mu^{+} \mu^{-}$and $\tau^{+} \tau^{-}$, are not strong enough to exclude any region of interest in our model parameter space. 


\section{Conclusions}

In this research, we work on a DM model with two gauge singlet fermionic WIMPs interacting with the SM particles via a Higgs portal. In fact, a singlet scalar mediator mixes with the SM Higgs and the interaction of the dark sector with the SM particles becomes possible. The light partner is stable and is the DM candidate and the heavy partner is a short-lived particle without contribution in the relic density.

It is found that the singlet fermionic DM model is excluded by the recent direct detection experiments, except in the resonance region. This motivates us to extend the minimal singlet DM model to incorporate two WIMPs instead of one. The DM relic density in our model involves contributions from annihilation and coannihilation processes. It turns out the coannihilation effects enhance the relic density.

We realized that even in the regions of the parameter space with suppressed coannihilation effects, the model shows some characteristic features when imposing both DM relic density constraints and direct detection bounds. In fact we found that there exist regions of the parameter space beyond the resonance region which evade direct detection upper limits. These new features arise from a Feynman diagram in the DM annihilation processes with an intermediate heavy WIMP which comes in due to its coupling to the DM candidate. We find that the size of the viable region depends mainly on the singlet scalar mass and the coannihilation effects.

Moreover, the constraints from Fermi-LAT upper limits on the DM annihilation into $W^{+} W^{-}$and $b \bar{b}$ are imposed on the viable parameter space. It turned out that only constraints from $b \bar{b}$ channel are strong enough, and can exclude a small portion of the constrained parameter space which escape the direct detection experiments.

Further works in this model suggest the search for the mono-X signatures in association with dark matter pair production at the LHC, for recent reviews see [37-39]. In this direction, a detailed study on various simplified DM models compares the reach of the mono-X searches and direct searches for the dark mediating particle [40].

\section{Acknowledgments}

The author wishes to thank the CERN theoretical physics department for the hospitality and support where this work was finalized during the visit. Arak University is acknowledged for financial support under contract no. 1397/750.

\section{A Annihilation cross sections}

In this section we provide the DM annihilation cross sections with two particles in the final state. The cross section with SM fermions in the final state is

$$
\begin{aligned}
\sigma v_{\text {rel }}\left(\bar{\psi}_{1} \psi_{1} \rightarrow \bar{f} f\right)= & \frac{N_{c} m_{f}^{2} g_{1}^{2} \sin ^{2} 2 \theta}{8 \pi s v_{h}^{2}}\left[\left(p_{1} \cdot p_{2}\right)^{2}-2\left(p_{1} \cdot p_{2}\right) m_{f}^{2}+2 m_{f}^{2} m_{1}^{2}-m_{1}^{4}\right] \\
& \times\left|\frac{1}{s-m_{s}^{2}+i m_{s} \Gamma_{s}}-\frac{1}{s-m_{h}^{2}+i m_{h} \Gamma_{h}}\right|^{2}
\end{aligned}
$$


where $N_{c}$ is the number of color. DM annihilation to a pair of $\mathrm{Z}$ boson is

$$
\begin{aligned}
& \sigma v_{\text {rel }}\left(\bar{\psi}_{1} \psi_{1} \rightarrow Z Z\right)=\frac{g_{1}^{2} \sin ^{2} 2 \theta}{16 \pi s v_{h}^{2}}\left[\left(p_{1} \cdot p_{2}\right)^{3}-2\left(p_{1} \cdot p_{2}\right)^{2} m_{Z}^{2}+\left(p_{1} \cdot p_{2}\right)^{2} m_{1}^{2}+2\left(p_{1} \cdot p_{2}\right) m_{Z}^{4}\right. \\
& \left.-\left(p_{1} \cdot p_{2}\right) m_{1}^{4}-3 m_{Z}^{4} m_{1}^{2}+2 m_{Z}^{2} m_{1}^{4}-m_{1}^{6}\right] \times\left|\frac{1}{s-m_{s}^{2}+i m_{s} \Gamma_{s}}-\frac{1}{s-m_{h}^{2}+i m_{h} \Gamma_{h}}\right|^{2}
\end{aligned}
$$

and to a pair of $\mathrm{W}$ boson is

$$
\begin{gathered}
\sigma v_{\mathrm{rel}}\left(\bar{\psi}_{1} \psi_{1} \rightarrow W^{+} W^{-}\right)=\frac{g_{1}^{2} \sin ^{2} 2 \theta}{8 \pi s v_{h}^{2}}\left[\left(p_{1} \cdot p_{2}\right)^{3}-2\left(p_{1} \cdot p_{2}\right)^{2} m_{W}^{2}+\left(p_{1} \cdot p_{2}\right)^{2} m_{1}^{2}+2\left(p_{1} \cdot p_{2}\right) m_{W}^{4}\right. \\
\left.-\left(p_{1} \cdot p_{2}\right) m_{1}^{4}-3 m_{W}^{4} m_{1}^{2}+2 m_{W}^{2} m_{1}^{4}-m_{1}^{6}\right] \times\left|\frac{1}{s-m_{s}^{2}+i m_{s} \Gamma_{s}}-\frac{1}{s-m_{h}^{2}+i m_{h} \Gamma_{h}}\right|^{2} .
\end{gathered}
$$

DM annihilation to a pair of singlet scalar is given by

$$
\begin{aligned}
& \sigma v_{\text {rel }}\left(\bar{\psi}_{1} \psi_{1} \rightarrow s s\right)=\frac{\sqrt{1-4 m_{h}^{2} / s}}{32 \pi^{2} s} \int d \Omega\left[\frac{1}{8} b_{1}^{2} \frac{g_{1}^{2} \sin ^{2} \theta\left[p_{1} \cdot p_{2}-m_{1}^{2}\right]}{\left(s-m_{h}^{2}\right)^{2}}+\frac{3}{4} b b_{1} \frac{g_{1}^{2} \sin ^{2} \theta \cos \theta\left[p_{1} \cdot p_{2}-m_{1}^{2}\right]}{\left(s-m_{s}^{2}\right)\left(s-m_{h}^{2}\right)}\right. \\
& -3 b \frac{g_{1} g_{12}^{2} \sin \theta \cos ^{3} \theta\left[2\left(p_{1} . p_{3}\right) m_{1}-\left(p_{1} . p_{2}\right) m_{2}-2\left(p_{1} \cdot p_{2}\right) m_{1}+m_{2} m_{1}^{2}\right]}{\left(s-m_{s}^{2}\right)\left(u-m_{2}^{2}\right)} \\
& +\frac{9}{8} b^{2} \frac{g_{1}^{2} \cos ^{2} \theta \sin ^{2} \theta\left[p_{1} \cdot p_{2}-m_{1}^{2}\right]}{\left(s-m_{s}^{2}\right)^{2}}+b_{1} \frac{g_{1} g_{12}^{2} \sin \theta \cos ^{2} \theta\left[2\left(p_{1} \cdot p_{3}\right) m_{1}+\left(p_{1} \cdot p_{2}\right) m_{2}-m_{2} m_{1}^{2}-2 m_{1}^{3}\right]}{\left(s-m_{h}^{2}\right)\left(t-m_{2}^{2}\right)} \\
& +b_{1} \frac{g_{1}^{3} m_{1} \sin \theta \cos ^{2} \theta\left[2\left(p_{1} \cdot p_{3}\right)+\left(p_{1} \cdot p_{2}\right)-3 m_{1}^{2}\right]}{\left(s-m_{h}^{2}\right)\left(t-m_{1}^{2}\right)}+3 b \frac{g_{1}^{3} m_{1} \sin \theta \cos ^{3} \theta\left[2\left(p_{1} \cdot p_{3}\right)+\left(p_{1} \cdot p_{2}\right)-3 m_{1}^{2}\right]}{\left(s-m_{s}^{2}\right)\left(t-m_{1}^{2}\right)} \\
& -g_{1}^{4} \cos ^{4} \theta \frac{\left[2\left(p_{1} \cdot p_{3}\right)^{2}-2\left(p_{1} \cdot p_{3}\right)\left(p_{1} \cdot p_{2}\right)-10\left(p_{1} \cdot p_{3}\right) m_{1}^{2}+\left(p_{1} \cdot p_{2}\right) m_{s}^{2}+m_{s}^{2} m_{1}^{2}+8 m_{1}^{4}\right]}{\left(t-m_{1}^{2}\right)^{2}} \\
& +g_{1}^{4} \cos ^{4} \theta \frac{\left[2\left(p_{1} \cdot p_{3}\right)^{2}-2\left(p_{1} \cdot p_{3}\right)\left(p_{1} \cdot p_{2}\right)-2\left(p_{1} \cdot p_{3}\right) m_{1}^{2}+\left(p_{1} \cdot p_{2}\right) m_{s}^{2}+4\left(p_{1} \cdot p_{2}\right) m_{1}^{2}+m_{s}^{2} m_{1}^{2}-4 m_{1}^{4}\right]}{\left(t-m_{1}^{2}\right)\left(u-m_{1}^{2}\right)} \\
& -2 \frac{g_{1}^{2} g_{12}^{2} \cos ^{4} \theta}{\left(t-m_{1}^{2}\right)\left(t-m_{2}^{2}\right)}\left[2\left(p_{1} \cdot p_{3}\right)^{2}-2\left(p_{1} \cdot p_{3}\right)\left(p_{1} \cdot p_{2}\right)-2\left(p_{1} \cdot p_{3}\right) m_{1} m_{2}-8\left(p_{1} \cdot p_{3}\right) m_{1}^{2}+\left(p_{1} \cdot p_{2}\right) m_{s}^{2}\right. \\
& \left.-\left(p_{1} \cdot p_{2}\right) m_{1} m_{2}+\left(p_{1} \cdot p_{2}\right) m_{1}^{2}+m_{s}^{2} m_{1}^{2}+3 m_{2} m_{1}^{3}+5 m_{1}^{4}\right] \\
& +2 \frac{g_{1}^{2} g_{12}^{2} \cos ^{4} \theta}{\left(t-m_{1}^{2}\right)\left(u-m_{2}^{2}\right)}\left[2\left(p_{1} \cdot p_{3}\right)^{2}-2\left(p_{1} \cdot p_{3}\right)\left(p_{1} \cdot p_{2}\right)+2\left(p_{1} \cdot p_{3}\right) m_{1} m_{2}-4\left(p_{1} \cdot p_{3}\right) m_{1}^{2}+\left(p_{1} \cdot p_{2}\right) m_{s}^{2}\right. \\
& \left.+\left(p_{1} \cdot p_{2}\right) m_{1} m_{2}+3\left(p_{1} \cdot p_{2}\right) m_{1}^{2}+m_{s}^{2} m_{1}^{2}-3 m_{2} m_{1}^{3}-m_{1}^{4}\right] \\
& -\frac{g_{12}^{4} \cos ^{4} \theta}{\left(t-m_{2}^{2}\right)^{2}}\left[2\left(p_{1} \cdot p_{3}\right)^{2}-2\left(p_{1} \cdot p_{3}\right)\left(p_{1} \cdot p_{2}\right)-4\left(p_{1} \cdot p_{3}\right) m_{1} m_{2}-6\left(p_{1} \cdot p_{3}\right) m_{1}^{2}+\left(p_{1} \cdot p_{2}\right) m_{s}^{2}\right. \\
& \left.-\left(p_{1} \cdot p_{2}\right) m_{2}^{2}+\left(p_{1} \cdot p_{2}\right) m_{1}^{2}+m_{s}^{2} m_{1}^{2}+m_{1}^{2} m_{2}^{2}+4 m_{2} m_{1}^{3}+3 m_{1}^{4}\right] \\
& +\frac{g_{12}^{4} \cos ^{4} \theta}{\left(t-m_{2}^{2}\right)\left(u-m_{2}^{2}\right)}\left[2\left(p_{1} \cdot p_{3}\right)^{2}-2\left(p_{1} \cdot p_{3}\right)\left(p_{1} \cdot p_{2}\right)-2\left(p_{1} \cdot p_{3}\right) m_{1}^{2}+\left(p_{1} \cdot p_{2}\right) m_{s}^{2}\right. \\
& \left.\left.+\left(p_{1} . p_{2}\right) m_{2}^{2}+2\left(p_{1} . p_{2}\right) m_{1} m_{2}+\left(p_{1} . p_{2}\right) m_{1}^{2}+m_{s}^{2} m_{1}^{2}-m_{1}^{2} m_{2}^{2}-2 m_{2} m_{1}^{3}-m_{1}^{4}\right]\right],
\end{aligned}
$$


where

$$
\begin{aligned}
b & =\sin \theta \cos \theta \lambda_{1}-2 \cos ^{2} \theta \lambda_{2} v_{h}-\sin ^{2} \theta \lambda_{H} v_{h}, \\
b_{1} & =3 \sin ^{3} \theta \lambda_{1}-2 \sin \theta \lambda_{1}-6 \cos \theta \sin ^{2} \theta \lambda_{2} v_{h}+2 \cos \theta \lambda_{2} v_{h}+3 \cos \theta \sin ^{2} \theta \lambda_{H} v_{h} .
\end{aligned}
$$

The rerevant annihilation cross section to a pair of Higgs boson is

$$
\begin{aligned}
& \sigma v_{\text {rel }}\left(\bar{\psi}_{1} \psi_{1} \rightarrow h h\right)=\frac{\sqrt{1-4 m_{h}^{2} / s}}{32 \pi^{2} s} \int d \Omega\left[\frac{1}{8} b_{2}^{2} \frac{g_{1}^{2} \cos ^{2} \theta\left[p_{1} \cdot p_{2}-m_{1}^{2}\right]}{\left(s-m_{s}^{2}\right)^{2}}-\frac{3}{4} b_{3} b_{2} \frac{g_{1}^{2} \cos ^{2} \theta \sin \theta\left[p_{1} \cdot p_{2}-m_{1}^{2}\right]}{\left(s-m_{s}^{2}\right)\left(s-m_{h}^{2}\right)}\right. \\
& +b_{2} m_{1} \frac{g_{1}^{3} \cos \theta \sin ^{2} \theta\left[2 p_{1} \cdot p_{3}-3 p_{1} \cdot p_{2}+m_{1}^{2}\right]}{\left(s-m_{s}^{2}\right)\left(u-m_{1}^{2}\right)} \\
& +b_{2} \frac{g_{1} g_{12}^{2} \cos \theta \sin ^{2} \theta\left[2\left(p_{1} \cdot p_{3}\right) m_{1}-\left(p_{1} \cdot p_{2}\right) m_{2}-2\left(p_{1} \cdot p_{2}\right) m_{1}+m_{2} m_{1}^{2}\right]}{\left(s-m_{s}^{2}\right)\left(u-m_{2}^{2}\right)} \\
& +\frac{9}{8} b_{3}^{2} \frac{g_{1}^{2} \cos ^{2} \theta \sin ^{2} \theta\left[p_{1} \cdot p_{2}-m_{1}^{2}\right]}{\left(s-m_{h}^{2}\right)^{2}}-3 b_{3} m_{1} \frac{g_{1}^{3} \cos \theta \sin ^{3} \theta\left[2 p_{1} \cdot p_{3}-3 p_{1} \cdot p_{2}+m_{1}^{2}\right]}{\left(s-m_{h}^{2}\right)\left(u-m_{1}^{2}\right)} \\
& -3 b_{3} \frac{g_{1} g_{12}^{2} \cos \theta \sin ^{3} \theta\left[2\left(p_{1} \cdot p_{3}\right) m_{1}-\left(p_{1} \cdot p_{2}\right) m_{2}-2\left(p_{1} \cdot p_{2}\right) m_{1}+m_{2} m_{1}^{2}\right]}{\left(s-m_{h}^{2}\right)\left(u-m_{2}^{2}\right)} \\
& -g_{1}^{4} \sin ^{4} \theta \frac{\left[2\left(p_{1} \cdot p_{3}\right)^{2}-2\left(p_{1} \cdot p_{3}\right)\left(p_{1} \cdot p_{2}\right)-10\left(p_{1} \cdot p_{3}\right) m_{1}^{2}+\left(p_{1} \cdot p_{2}\right) m_{h}^{2}+m_{h}^{2} m_{1}^{2}+8 m_{1}^{4}\right]}{\left(t-m_{1}^{2}\right)^{2}} \\
& +g_{1}^{4} \sin ^{4} \theta \frac{\left[2\left(p_{1} \cdot p_{3}\right)^{2}-2\left(p_{1} \cdot p_{3}\right)\left(p_{1} \cdot p_{2}\right)-2\left(p_{1} \cdot p_{3}\right) m_{1}^{2}+\left(p_{1} \cdot p_{2}\right) m_{h}^{2}+4\left(p_{1} \cdot p_{2}\right) m_{1}^{2}+m_{h}^{2} m_{1}^{2}-4 m_{1}^{4}\right]}{\left(t-m_{1}^{2}\right)\left(u-m_{1}^{2}\right)} \\
& -2 \frac{g_{1}^{2} g_{12}^{2} \sin ^{4} \theta}{\left(t-m_{1}^{2}\right)\left(t-m_{2}^{2}\right)}\left[2\left(p_{1} \cdot p_{3}\right)^{2}-2\left(p_{1} \cdot p_{3}\right)\left(p_{1} \cdot p_{2}\right)-2\left(p_{1} \cdot p_{3}\right) m_{1} m_{2}-8\left(p_{1} \cdot p_{3}\right) m_{1}^{2}+\left(p_{1} \cdot p_{2}\right) m_{h}^{2}\right. \\
& \left.-\left(p_{1} \cdot p_{2}\right) m_{1} m_{2}+\left(p_{1} \cdot p_{2}\right) m_{1}^{2}+m_{h}^{2} m_{1}^{2}+3 m_{2} m_{1}^{3}+5 m_{1}^{4}\right] \\
& +2 \frac{g_{1}^{2} g_{12}^{2} \sin ^{4} \theta}{\left(t-m_{1}^{2}\right)\left(u-m_{2}^{2}\right)}\left[2\left(p_{1} \cdot p_{3}\right)^{2}-2\left(p_{1} \cdot p_{3}\right)\left(p_{1} \cdot p_{2}\right)+2\left(p_{1} \cdot p_{3}\right) m_{1} m_{2}-4\left(p_{1} \cdot p_{3}\right) m_{1}^{2}+\left(p_{1} \cdot p_{2}\right) m_{h}^{2}\right. \\
& \left.+\left(p_{1} \cdot p_{2}\right) m_{1} m_{2}+3\left(p_{1} \cdot p_{2}\right) m_{1}^{2}+m_{h}^{2} m_{1}^{2}-3 m_{2} m_{1}^{3}-m_{1}^{4}\right] \\
& -\frac{g_{12}^{4} \sin ^{4} \theta}{\left(t-m_{2}^{2}\right)^{2}}\left[2\left(p_{1} \cdot p_{3}\right)^{2}-2\left(p_{1} \cdot p_{3}\right)\left(p_{1} \cdot p_{2}\right)-4\left(p_{1} \cdot p_{3}\right) m_{1} m_{2}-6\left(p_{1} \cdot p_{3}\right) m_{1}^{2}+\left(p_{1} \cdot p_{2}\right) m_{h}^{2}\right. \\
& \left.-\left(p_{1} \cdot p_{2}\right) m_{2}^{2}+\left(p_{1} \cdot p_{2}\right) m_{1}^{2}+m_{h}^{2} m_{1}^{2}+m_{1}^{2} m_{2}^{2}+4 m_{2} m_{1}^{3}+3 m_{1}^{4}\right] \\
& +\frac{g_{12}^{4} \sin ^{4} \theta}{\left(t-m_{2}^{2}\right)\left(u-m_{2}^{2}\right)}\left[2\left(p_{1} \cdot p_{3}\right)^{2}-2\left(p_{1} \cdot p_{3}\right)\left(p_{1} \cdot p_{2}\right)-2\left(p_{1} \cdot p_{3}\right) m_{1}^{2}+\left(p_{1} \cdot p_{2}\right) m_{h}^{2}\right. \\
& \left.\left.+\left(p_{1} . p_{2}\right) m_{2}^{2}+2\left(p_{1} \cdot p_{2}\right) m_{1} m_{2}+\left(p_{1} . p_{2}\right) m_{1}^{2}+m_{h}^{2} m_{1}^{2}-m_{1}^{2} m_{2}^{2}-2 m_{2} m_{1}^{3}-m_{1}^{4}\right]\right],
\end{aligned}
$$

where

$$
\begin{aligned}
& b_{2}=3 \lambda_{1} \cos \theta \sin ^{2} \theta-\lambda_{1} \cos \theta+6 \lambda_{2} v_{h} \sin ^{3} \theta-4 \lambda_{2} v_{h} \sin \theta+3 \lambda_{H} v_{h} \cos ^{2} \theta \sin \theta, \\
& b_{3}=\lambda_{1} \cos \theta \sin \theta+2 \lambda_{2} v_{h} \sin ^{2} \theta+\lambda_{H} v_{h} \cos ^{2} \theta .
\end{aligned}
$$


Finally, the DM annihilation to a singlet scalar and SM Higgs boson is

$$
\begin{aligned}
& \sigma v_{\text {rel }}\left(\bar{\psi}_{1} \psi_{1} \rightarrow h s\right)=\frac{\sqrt{1-4 m_{h}^{2} / s}}{32 \pi^{2} s} \int d \Omega\left[\frac{1}{4} b_{1}^{2} \frac{g_{1}^{2} \cos ^{2} \theta\left[p_{1} \cdot p_{2}-m_{1}^{2}\right]}{\left(s-m_{s}^{2}\right)^{2}}+\frac{1}{4} b_{2}^{2} \frac{g_{1}^{2} \sin ^{2} \theta\left[p_{1} \cdot p_{2}-m_{1}^{2}\right]}{\left(s-m_{h}^{2}\right)^{2}}\right. \\
& -\frac{1}{2} b_{1} b_{2} \frac{g_{1}^{2} \cos \theta \sin \theta\left[p_{1} \cdot p_{2}-m_{1}^{2}\right]}{\left(s-m_{s}^{2}\right)\left(s-m_{h}^{2}\right)}+\frac{1}{2} b_{1} m_{1} \frac{g_{1}^{3} \cos ^{2} \theta \sin \theta\left[4 p_{1} \cdot p_{3}+2 p_{1} \cdot p_{2}+m_{h}^{2}-6 m_{1}^{2}-m_{s}^{2}\right]}{\left(s-m_{s}^{2}\right)\left(t-m_{1}^{2}\right)} \\
& -\frac{1}{2} b_{2} m_{1} \frac{g_{1}^{3} \cos \theta \sin ^{2} \theta\left[4 p_{1} . p_{3}+2 p_{1} . p_{2}+m_{h}^{2}-6 m_{1}^{2}-m_{s}^{2}\right]}{\left(s-m_{h}^{2}\right)\left(t-m_{1}^{2}\right)} \\
& -\frac{1}{2} b_{1} m_{1} \frac{g_{1}^{3} \cos ^{2} \theta \sin \theta\left[4 p_{1} \cdot p_{3}-6 p_{1} \cdot p_{2}+m_{h}^{2}+2 m_{1}^{2}-m_{s}^{2}\right]}{\left(s-m_{s}^{2}\right)\left(u-m_{1}^{2}\right)} \\
& +\frac{1}{2} b_{2} m_{1} \frac{g_{1}^{3} \cos \theta \sin ^{2} \theta\left[4 p_{1} \cdot p_{3}-6 p_{1} \cdot p_{2}+m_{h}^{2}+2 m_{1}^{2}-m_{s}^{2}\right]}{\left(s-m_{h}^{2}\right)\left(u-m_{1}^{2}\right)} \\
& +\frac{1}{2} b_{1} \frac{g_{1} g_{12}^{2} \cos ^{2} \theta \sin \theta\left[4\left(p_{1} . p_{3}\right) m_{1}+2\left(p_{1} . p_{2}\right) m_{2}+m_{h}^{2} m_{1}-2 m_{1}^{2} m_{2}-4 m_{1}^{3}-m_{1} m_{s}^{2}\right]}{\left(s-m_{s}^{2}\right)\left(t-m_{2}^{2}\right)} \\
& -\frac{1}{2} b_{2} \frac{g_{1} g_{12}^{2} \cos \theta \sin ^{2} \theta\left[4\left(p_{1} . p_{3}\right) m_{1}+2\left(p_{1} . p_{2}\right) m_{2}+m_{h}^{2} m_{1}-2 m_{1}^{2} m_{2}-4 m_{1}^{3}-m_{1} m_{s}^{2}\right]}{\left(s-m_{h}^{2}\right)\left(t-m_{2}^{2}\right)} \\
& +\frac{1}{2} b_{2} \frac{g_{1} g_{12}^{2} \cos \theta \sin ^{2} \theta\left[4\left(p_{1} \cdot p_{3}\right) m_{1}-2\left(p_{1} \cdot p_{2}\right) m_{2}-4\left(p_{1} \cdot p_{2}\right) m_{1}+m_{h}^{2} m_{1}+2 m_{1}^{2} m_{2}-m_{1} m_{s}^{2}\right]}{\left(s-m_{h}^{2}\right)\left(u-m_{2}^{2}\right)} \\
& -\frac{g_{1}^{4} \sin ^{2} \theta \cos ^{2} \theta}{\left(t-m_{1}^{2}\right)^{2}}\left[2\left(p_{1} \cdot p_{3}\right)^{2}-2\left(p_{1} \cdot p_{3}\right)\left(p_{1} \cdot p_{2}\right)-10\left(p_{1} . p_{3}\right) m_{1}^{2}+\left(p_{1} \cdot p_{3}\right) m_{h}^{2}-\left(p_{1} \cdot p_{3}\right) m_{s}^{2}\right. \\
& \left.+\left(p_{1} \cdot p_{2}\right) m_{s}^{2}-2 m_{h}^{2} m_{1}^{2}+8 m_{1}^{4}+3 m_{1}^{2} m_{s}^{2}\right] \\
& -\frac{g_{1}^{4} \sin ^{2} \theta \cos ^{2} \theta}{\left(u-m_{1}^{2}\right)^{2}}\left[2\left(p_{1} \cdot p_{3}\right)^{2}-2\left(p_{1} \cdot p_{3}\right)\left(p_{1} \cdot p_{2}\right)+6\left(p_{1} \cdot p_{3}\right) m_{1}^{2}+\left(p_{1} \cdot p_{3}\right) m_{h}^{2}-\left(p_{1} \cdot p_{3}\right) m_{s}^{2}\right. \\
& \left.+\left(p_{1} \cdot p_{2}\right) m_{s}^{2}-8\left(p_{1} \cdot p_{2}\right) m_{1}^{2}+2 m_{h}^{2} m_{1}^{2}-m_{1}^{2} m_{s}^{2}\right] \\
& +2 \frac{g_{1}^{4} \sin ^{2} \theta \cos ^{2} \theta}{\left(t-m_{1}^{2}\right)\left(u-m_{1}^{2}\right)}\left[2\left(p_{1} \cdot p_{3}\right)^{2}-2\left(p_{1} \cdot p_{3}\right)\left(p_{1} \cdot p_{2}\right)-2\left(p_{1} \cdot p_{3}\right) m_{1}^{2}+\left(p_{1} \cdot p_{3}\right) m_{h}^{2}\right. \\
& \left.-\left(p_{1} \cdot p_{3}\right) m_{s}^{2}+\left(p_{1} \cdot p_{2}\right) m_{s}^{2}+4\left(p_{1} \cdot p_{2}\right) m_{1}^{2}-4 m_{1}^{4}+m_{1}^{2} m_{s}^{2}\right] \\
& +\frac{g_{1}^{2} g_{12}^{2} \sin ^{2} \theta \cos ^{2} \theta}{\left(t-m_{2}^{2}\right)\left(u-m_{1}^{2}\right)}\left[4\left(p_{1} \cdot p_{3}\right)^{2}-4\left(p_{1} \cdot p_{3}\right)\left(p_{1} \cdot p_{2}\right)-4\left(p_{1} \cdot p_{3}\right) m_{1} m_{2}+2\left(p_{1} \cdot p_{3}\right) m_{h}^{2}\right. \\
& -2\left(p_{1} \cdot p_{3}\right) m_{s}^{2}+2\left(p_{1} \cdot p_{2}\right) m_{s}^{2}+6\left(p_{1} . p_{2}\right) m_{1} m_{2}+2\left(p_{1} . p_{2}\right) m_{1}^{2}-m_{h}^{2} m_{1} m_{2} \\
& \left.+m_{h}^{2} m_{1}^{2}-2 m_{2} m_{1}^{3}+m_{2} m_{1} m_{s}^{2}-6 m_{1}^{4}+m_{1}^{2} m_{s}^{2}\right] \\
& -\frac{g_{1}^{2} g_{12}^{2} \sin ^{2} \theta \cos ^{2} \theta}{\left(t-m_{1}^{2}\right)\left(t-m_{2}^{2}\right)}\left[4\left(p_{1} \cdot p_{3}\right)^{2}-4\left(p_{1} \cdot p_{3}\right)\left(p_{1} \cdot p_{2}\right)-4\left(p_{1} \cdot p_{3}\right) m_{1} m_{2}+2\left(p_{1} \cdot p_{3}\right) m_{h}^{2}-16\left(p_{1} \cdot p_{3}\right) m_{1}^{2}\right. \\
& -2\left(p_{1} \cdot p_{3}\right) m_{s}^{2}-2\left(p_{1} \cdot p_{2}\right) m_{1} m_{2}+2\left(p_{1} \cdot p_{2}\right) m_{1}^{2}+2\left(p_{1} \cdot p_{2}\right) m_{s}^{2}-m_{h}^{2} m_{1} m_{2} \\
& \left.-3 m_{h}^{2} m_{1}^{2}+6 m_{2} m_{1}^{3}+m_{2} m_{1} m_{s}^{2}+10 m_{1}^{4}+5 m_{1}^{2} m_{s}^{2}\right] \\
& +\frac{g_{1}^{2} g_{12}^{2} \sin ^{2} \theta \cos ^{2} \theta}{\left(t-m_{1}^{2}\right)\left(u-m_{2}^{2}\right)}\left[4\left(p_{1} \cdot p_{3}\right)^{2}-4\left(p_{1} \cdot p_{3}\right)\left(p_{1} \cdot p_{2}\right)+4\left(p_{1} \cdot p_{3}\right) m_{1} m_{2}+2\left(p_{1} \cdot p_{3}\right) m_{h}^{2}\right. \\
& -8\left(p_{1} \cdot p_{3}\right) m_{1}^{2}-2\left(p_{1} \cdot p_{3}\right) m_{s}^{2}+2\left(p_{1} \cdot p_{2}\right) m_{1} m_{2}+6\left(p_{1} \cdot p_{2}\right) m_{1}^{2}+2\left(p_{1} \cdot p_{2}\right) m_{s}^{2} \\
& \left.+m_{h}^{2} m_{1} m_{2}-m_{h}^{2} m_{1}^{2}-6 m_{2} m_{1}^{3}-m_{2} m_{1} m_{s}^{2}-2 m_{1}^{4}+3 m_{1}^{2} m_{s}^{2}\right] \\
& -\frac{g_{1}^{2} g_{12}^{2} \sin ^{2} \theta \cos ^{2} \theta}{\left(u-m_{1}^{2}\right)\left(u-m_{2}^{2}\right)}\left[4\left(p_{1} \cdot p_{3}\right)^{2}-4\left(p_{1} \cdot p_{3}\right)\left(p_{1} \cdot p_{2}\right)+4\left(p_{1} \cdot p_{3}\right) m_{1} m_{2}+2\left(p_{1} \cdot p_{3}\right) m_{h}^{2}\right. \\
& +8\left(p_{1} \cdot p_{3}\right) m_{1}^{2}-2\left(p_{1} \cdot p_{3}\right) m_{s}^{2}-6\left(p_{1} \cdot p_{2}\right) m_{1} m_{2}-10\left(p_{1} \cdot p_{2}\right) m_{1}^{2}+2\left(p_{1} \cdot p_{2}\right) m_{s}^{2} \\
& \left.+m_{h}^{2} m_{1} m_{2}+3 m_{h}^{2} m_{1}^{2}+2 m_{2} m_{1}^{3}-m_{2} m_{1} m_{s}^{2}-2 m_{1}^{4}-m_{1}^{2} m_{s}^{2}\right]
\end{aligned}
$$




$$
\begin{aligned}
& -\frac{g_{12}^{4} \sin ^{2} \theta \cos ^{2} \theta}{\left(t-m_{2}^{2}\right)^{2}}\left[2\left(p_{1} \cdot p_{3}\right)^{2}-2\left(p_{1} \cdot p_{3}\right)\left(p_{1} \cdot p_{2}\right)-4\left(p_{1} \cdot p_{3}\right) m_{1} m_{2}+\left(p_{1} \cdot p_{3}\right) m_{h}^{2}\right. \\
& -6\left(p_{1} \cdot p_{3}\right) m_{1}^{2}-\left(p_{1} \cdot p_{3}\right) m_{s}^{2}-\left(p_{1} \cdot p_{3}\right) m_{s}^{2}-\left(p_{1} \cdot p_{2}\right) m_{2}^{2}+\left(p_{1} \cdot p_{2}\right) m_{1}^{2} \\
& +\left(p_{1} \cdot p_{2}\right) m_{s}^{2}-m_{h}^{2} m_{1} m_{2}-m_{h}^{2} m_{1}^{2}+m_{2}^{2} m_{1}^{2}+4 m_{2} m_{1}^{3}+m_{2} m_{1} m_{s}^{2} \\
& \left.+3 m_{1}^{4}+2 m_{1}^{2} m_{s}^{2}\right] \\
& +2 \frac{g_{12}^{4} \sin ^{2} \theta \cos ^{2} \theta}{\left(t-m_{2}^{2}\right)\left(u-m_{2}^{2}\right)}\left[2\left(p_{1} \cdot p_{3}\right)^{2}-2\left(p_{1} \cdot p_{3}\right)\left(p_{1} \cdot p_{2}\right)+\left(p_{1} \cdot p_{3}\right) m_{h}^{2}-2\left(p_{1} \cdot p_{3}\right) m_{1}^{2}\right. \\
& -\left(p_{1} \cdot p_{3}\right) m_{s}^{2}+\left(p_{1} \cdot p_{2}\right) m_{s}^{2}+\left(p_{1} \cdot p_{2}\right) m_{2}^{2}+\left(p_{1} \cdot p_{2}\right) m_{1}^{2}+2\left(p_{1} \cdot p_{2}\right) m_{1} m_{2} \\
& \left.-m_{2}^{2} m_{1}^{2}-2 m_{2} m_{1}^{3}-m_{1}^{4}+m_{1}^{2} m_{s}^{2}\right] \\
& -\frac{g_{12}^{4} \sin ^{2} \theta \cos ^{2} \theta}{\left(u-m_{2}^{2}\right)^{2}}\left[2\left(p_{1} \cdot p_{3}\right)^{2}-2\left(p_{1} \cdot p_{3}\right)\left(p_{1} \cdot p_{2}\right)+4\left(p_{1} \cdot p_{3}\right) m_{1} m_{2}-4\left(p_{1} \cdot p_{2}\right) m_{1} m_{2}\right. \\
& +\left(p_{1} \cdot p_{3}\right) m_{h}^{2}+2\left(p_{1} \cdot p_{3}\right) m_{1}^{2}-\left(p_{1} \cdot p_{3}\right) m_{s}^{2}+\left(p_{1} \cdot p_{2}\right) m_{s}^{2}-\left(p_{1} \cdot p_{2}\right) m_{2}^{2} \\
& \left.\left.-3\left(p_{1} . p_{2}\right) m_{1}^{2}+m_{h}^{2} m_{1} m_{2}+m_{h}^{2} m_{1}^{2}+m_{2}^{2} m_{1}^{2}-m_{2} m_{1} m_{s}^{2}-m_{1}^{4}\right]\right] \text {. }
\end{aligned}
$$

Open Access. This article is distributed under the terms of the Creative Commons Attribution License (CC-BY 4.0), which permits any use, distribution and reproduction in any medium, provided the original author(s) and source are credited.

\section{References}

[1] G. Bertone, D. Hooper and J. Silk, Particle dark matter: Evidence, candidates and constraints, Phys. Rept. 405 (2005) 279 [hep-ph/0404175] [INSPIRE].

[2] L. Bergstrom, Dark Matter Evidence, Particle Physics Candidates and Detection Methods, Annalen Phys. 524 (2012) 479 [arXiv: 1205.4882] [INSPIRE].

[3] WMAP collaboration, G. Hinshaw et al., Nine-Year Wilkinson Microwave Anisotropy Probe (WMAP) Observations: Cosmological Parameter Results, Astrophys. J. Suppl. 208 (2013) 19 [arXiv: 1212.5226] [INSPIRE].

[4] Planck collaboration, P.A.R. Ade et al., Planck 2013 results. XVI. Cosmological parameters, Astron. Astrophys. 571 (2014) A16 [arXiv:1303.5076] [INSPIRE].

[5] XENON collaboration, E. Aprile et al., First Dark Matter Search Results from the XENON1T Experiment, Phys. Rev. Lett. 119 (2017) 181301 [arXiv:1705.06655] [InSPIRE].

[6] LUX collaboration, D.S. Akerib et al., Results from a search for dark matter in the complete LUX exposure, Phys. Rev. Lett. 118 (2017) 021303 [arXiv: 1608.07648] [INSPIRE].

[7] LUX collaboration, D.S. Akerib et al., Limits on spin-dependent WIMP-nucleon cross section obtained from the complete LUX exposure, Phys. Rev. Lett. 118 (2017) 251302 [arXiv: 1705.03380] [INSPIRE].

[8] B.W. Lee and S. Weinberg, Cosmological Lower Bound on Heavy Neutrino Masses, Phys. Rev. Lett. 39 (1977) 165 [INSPIRE].

[9] ATLAS collaboration, Observation of a new particle in the search for the Standard Model Higgs boson with the ATLAS detector at the LHC, Phys. Lett. B 716 (2012) 1 [arXiv: 1207.7214] [INSPIRE]. 
[10] CMS collaboration, Observation of a new boson at a mass of $125 \mathrm{GeV}$ with the CMS experiment at the LHC, Phys. Lett. B 716 (2012) 30 [arXiv:1207.7235] [INSPIRE].

[11] G.F. Giudice, The Dawn of the Post-Naturalness Era, arXiv:1710.07663.

[12] Y.G. Kim, K.Y. Lee and S.-H. Nam, Singlet fermionic dark matter with Veltman conditions, Phys. Lett. B 782 (2018) 316 [arXiv:1801.04074] [INSPIRE].

[13] K. Kainulainen, K. Tuominen and J. Virkajärvi, A model for dark matter, naturalness and a complete gauge unification, JCAP 07 (2015) 034 [arXiv: 1504.07197] [INSPIRE].

[14] K. Ghorbani and H. Ghorbani, Scalar Dark Matter in Scale Invariant Standard Model, JHEP 04 (2016) 024 [arXiv: 1511.08432] [INSPIRE].

[15] E. Morgante, Simplified Dark Matter Models, arXiv:1804.01245 [INSPIRE].

[16] V. Silveira and A. Zee, Scalar phantoms, Phys. Lett. B 161 (1985) 136 [InSPIRE].

[17] J. McDonald, Gauge singlet scalars as cold dark matter, Phys. Rev. D 50 (1994) 3637 [hep-ph/0702143] [INSPIRE].

[18] C.P. Burgess, M. Pospelov and T. ter Veldhuis, The Minimal model of nonbaryonic dark matter: A Singlet scalar, Nucl. Phys. B 619 (2001) 709 [hep-ph/0011335] [INSPIRE].

[19] Y.G. Kim, K.Y. Lee and S. Shin, Singlet fermionic dark matter, JHEP 05 (2008) 100 [arXiv:0803.2932] [INSPIRE].

[20] K. Ghorbani, Fermionic dark matter with pseudo-scalar Yukawa interaction, JCAP 01 (2015) 015 [arXiv: 1408.4929] [INSPIRE].

[21] K. Ghorbani, Renormalization group equation analysis of a pseudoscalar portal dark matter model, J. Phys. G 44 (2017) 105006 [arXiv:1702.08711] [INSPIRE].

[22] GAMBIT collaboration, P. Athron et al., Status of the scalar singlet dark matter model, Eur. Phys. J. C 77 (2017) 568 [arXiv:1705.07931] [INSPIRE].

[23] M. Ettefaghi and R. Moazzemi, Analyzing of singlet fermionic dark matter via the updated direct detection data, Eur. Phys. J. C 77 (2017) 343 [arXiv:1705.07571] [INSPIRE].

[24] K. Ghorbani and H. Ghorbani, Scalar split WIMPs in future direct detection experiments, Phys. Rev. D 93 (2016) 055012 [arXiv: 1501.00206] [INSPIRE].

[25] S. Bhattacharya, P. Ghosh, T.N. Maity and T.S. Ray, Mitigating Direct Detection Bounds in Non-minimal Higgs Portal Scalar Dark Matter Models, JHEP 10 (2017) 088 [arXiv: 1706.04699] [INSPIRE].

[26] J.A. Casas, D.G. Cerdeño, J.M. Moreno and J. Quilis, Reopening the Higgs portal for single scalar dark matter, JHEP 05 (2017) 036 [arXiv: 1701.08134] [INSPIRE].

[27] A. Belyaev, N.D. Christensen and A. Pukhov, CalcHEP 3.4 for collider physics within and beyond the Standard Model, Comput. Phys. Commun. 184 (2013) 1729 [arXiv:1207.6082] [INSPIRE].

[28] K. Griest and D. Seckel, Three exceptions in the calculation of relic abundances, Phys. Rev. D 43 (1991) 3191 [INSPIRE].

[29] J. Edsjo and P. Gondolo, Neutralino relic density including coannihilations, Phys. Rev. D 56 (1997) 1879 [hep-ph/9704361] [INSPIRE].

[30] D. Barducci et al., Collider limits on new physics within MicrOMEGAs_4.3, Comput. Phys. Commun. 222 (2018) 327 [arXiv:1606.03834] [INSPIRE]. 
[31] J. Edsjo, M. Schelke, P. Ullio and P. Gondolo, Accurate relic densities with neutralino, chargino and sfermion coannihilations in mSUGRA, JCAP 04 (2003) 001 [hep-ph/0301106] [INSPIRE].

[32] S. Profumo and A. Provenza, Increasing the neutralino relic abundance with slepton coannihilations: Consequences for indirect dark matter detection, JCAP 12 (2006) 019 [hep-ph/0609290] [INSPIRE].

[33] J.R. Ellis, K.A. Olive and C. Savage, Hadronic Uncertainties in the Elastic Scattering of Supersymmetric Dark Matter, Phys. Rev. D 77 (2008) 065026 [arXiv:0801.3656] [InSPIRE].

[34] A. Crivellin, M. Hoferichter and M. Procura, Accurate evaluation of hadronic uncertainties in spin-independent WIMP-nucleon scattering: Disentangling two- and three-flavor effects, Phys. Rev. D 89 (2014) 054021 [arXiv:1312.4951] [INSPIRE].

[35] Fermi-LAT collaboration, M. Ackermann et al., Searching for Dark Matter Annihilation from Milky Way Dwarf Spheroidal Galaxies with Six Years of Fermi Large Area Telescope Data, Phys. Rev. Lett. 115 (2015) 231301 [arXiv: 1503.02641] [INSPIRE].

[36] H.E.S.S. collaboration, H. Abdallah et al., Search for dark matter annihilations towards the inner Galactic halo from 10 years of observations with H.E.S.S, Phys. Rev. Lett. 117 (2016) 111301 [arXiv: 1607.08142] [INSPIRE].

[37] F. Kahlhoefer, Review of LHC Dark Matter Searches, Int. J. Mod. Phys. A 32 (2017) 1730006 [arXiv: 1702.02430] [INSPIRE].

[38] B. Penning, The pursuit of dark matter at colliders - an overview, J. Phys. G 45 (2018) 063001 [arXiv: 1712.01391] [INSPIRE].

[39] T. Plehn, Yet Another Introduction to Dark Matter, arXiv:1705.01987 [INSPIRE].

[40] S.P. Liew, M. Papucci, A. Vichi and K.M. Zurek, Mono-X Versus Direct Searches: Simplified Models for Dark Matter at the LHC, JHEP 06 (2017) 082 [arXiv:1612.00219] [INSPIRE]. 